\title{
Real-time detection system for automobile car seat based on vision
}

\author{
Li Xiaoguang ${ }^{1, ~ a, ~ Z h u ~ J u a n ~}{ }^{2, \text { b, * } \text {, and Wang Chao }}{ }^{2, c}$ \\ ${ }^{1}$ Changchun Guanghua University, Changchun China \\ ${ }^{2}$ Institute of Electrical Information, Changchun Guanghua University, Changchun, China \\ a 14525451@qq.com, ${ }^{b}$ zhuj_guanghua@126.com, ${ }^{\mathrm{c}}$ 71128221@qq.com \\ *Corresponding author
}

Keywords: Car seat detection, machine vision, Camera calibration.

\begin{abstract}
Most car seat detection method is artificial, which has low efficiency. To solve this problem, proposed a real-time detection system based on vision in this paper. Firstly, give the total block diagram of the system. Secondly, give the camera calibration method. Third, use significant value to detect the springs and use the Hough transform to detect the side curtain. Calculate the intersections between springs and the side curtain. If the intersections are 8, the car seat is qualified.
\end{abstract}

\section{Introduction}

Auto parts for car quality have important influence on the overall performance. Auto parts in production and processing is completed, it is necessary to detect their quality. Car seat is one of auto parts. So it is necessary to detect the quality of car seat. The traditional inspection of car seat quality is mainly done by manual or auxiliary machines. The effect of manual detection is limited by the operating personnel's physical condition and working condition. The disadvantages of artificial detection appear. Negligence leads to the unqualified products into the subsequent production process, which caused a great deal of accidents.

In current automobile industry, the industry demand new requirements for the quality of automobile car seat. With this requirement, machine vision technology is introduced into auto parts testing. Machine vision detection technology uses industrial camera to simulate the eyes of people. Controller is used to simulate the human brain. Eventually detect the car seat to complete testing of products. The technology has the characteristics of non-contact, real time, high precision and easy to be automated. At the same time, the technology has greatly promoted the transformation of industrial production mode from labour intensive to technology-intensive.

\section{Structure of the machine vision detection system}

On the basis of the geometry size of product to be detected, surface properties and shape properties, such as machine vision detection system to deal with product's image, and to control the actuator according to the results of the treatment of complete the corresponding operation. The main process is:

\subsection{Image Acquisition}

This step involves the light source module, the image acquisition module. The selection and design of the module of the light source need to be determined according to the characteristics of the car seat and the detection target. Industrial camera image collection of artefacts, and the image to the controller, different applications for different controller, such as the embedded controller, such as industrial control computer, this system adopts industrial control computer.

\subsection{Image Processing}

Different testing requirements correspond to different ways of image processing, such as the size of the common measurement will involve the edge detection, defect detection will be according to 
the character of the defect has a different approach.

\subsection{Products classification}

The software can be used to evaluate the quality of the product, and output the signal to the actuator or other type of control system, the electrical control system (PLC), and perform the corresponding operation.

Block diagram of visual inspection system is shown in figure 1.

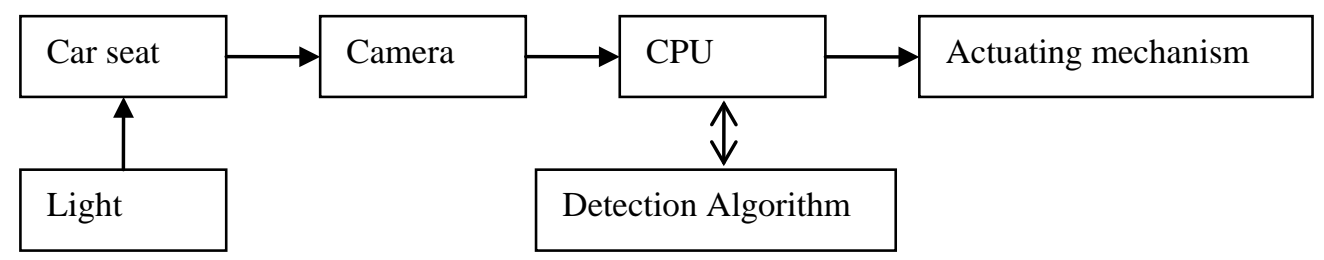

Figure 1 Block diagram of visual inspection system.

\section{Software of detection system}

Software of the visual system is to process the images obtained by industrial camera. Get the pixel information of car seat. Compare the detected car seat with qualified car seat. Determine whether the detected car seat is qualified or not. Give corresponding control signal to actuator.

\subsection{Camera calibration}

The visual measurement technology is based on the corresponding relationship between the actual target and the target image in the three-dimensional world, which requires the camera calibration to determine the corresponding parameters of the camera. The accuracy of visual measurement is also closely related to the camera calibration condition. Use needle-hole camera model to calibrate CCD surface array camera which is shown in figure 2.

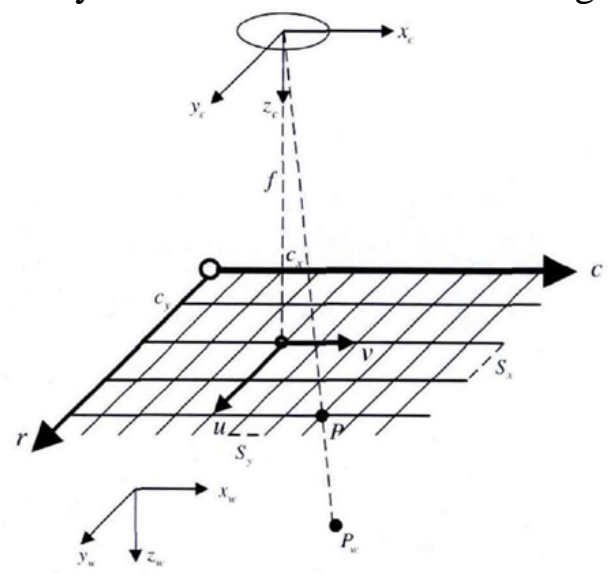

Figure 2 Diagram of the needle-hole camera model.

The relationship between the world coordinate system and the camera coordinate system is shown in formula 1.

$$
\left[\begin{array}{l}
x_{C} \\
y_{C} \\
z_{C}
\end{array}\right]=R\left[\begin{array}{c}
x_{W} \\
y_{W} \\
z_{W}
\end{array}\right]+T=\left[\begin{array}{lll}
r_{11} & r_{12} & r_{13} \\
r_{21} & r_{22} & r_{23} \\
r_{31} & r_{32} & r_{33}
\end{array}\right]\left[\begin{array}{c}
x_{W} \\
y_{W} \\
z_{W}
\end{array}\right]+\left[\begin{array}{c}
t_{1} \\
t_{2} \\
t_{3}
\end{array}\right]
$$

In formula $1,\left(x_{C}, y_{C}, z_{C}\right)$ is in the camera coordinate. $\left(x_{W}, y_{W}, z_{W}\right)$ is in the world coordinate. $R$ represents the rotation orthogonal matrix. $T$ represents the translation transformation matrix.

The camera coordinate model can be described as formula 2. 


$$
\left\{\begin{array}{l}
u=x / d_{x}+u_{0} \\
v=y / d_{y}+v_{0}
\end{array}\right.
$$

$(u, v)$ is the pixel of image coordinate. $(x, y)$ is the length of image coordinate. It can also be shown as formula 3.

$$
\left[\begin{array}{c}
u \\
V \\
1
\end{array}\right]=\left[\begin{array}{ccc}
\frac{1}{d_{x}} & 0 & u_{0} \\
0 & \frac{1}{d_{y}} & v_{0} \\
0 & 0 & 1
\end{array}\right]\left[\begin{array}{c}
x \\
y \\
1
\end{array}\right]
$$

The visual inspection system of this paper is relative distance. So you can move the origin of the world coordinate system to the origin of the camera coordinate system. That is $\mathrm{R}$ is $3 * 3$-unit matrix. $\mathrm{T}$ is $3^{*} 10$ matrix. $z_{C}$ equals to $z_{W}$. So the relationship between image coordinate and world coordinate is as formula 4 .

$$
\left[\begin{array}{c}
u \\
V \\
1
\end{array}\right]=\frac{1}{Z_{C}}\left[\begin{array}{cccc}
f_{x} & 0 & u_{0} & 0 \\
0 & f_{y} & v_{0} & 0 \\
0 & 0 & 1 & 0
\end{array}\right]\left[\begin{array}{cc}
I_{3 * 3} & 0_{3 * 1} \\
0 & 1
\end{array}\right]\left[\begin{array}{c}
x_{W} \\
y_{W} \\
z_{W} \\
1
\end{array}\right]=\frac{1}{Z_{C}}\left[\begin{array}{cccc}
f_{x} & 0 & u_{0} & 0 \\
0 & f_{y} & V_{0} & 0 \\
0 & 0 & 1 & 0
\end{array}\right]\left[\begin{array}{c}
x_{W} \\
y_{W} \\
z_{W} \\
1
\end{array}\right]
$$

The distance between target and the camera is fixed. So $z_{W}$ is a fixed value. So formula 4 can be recognized as formula 5 for short.

$$
\left[\begin{array}{l}
u \\
v
\end{array}\right]=\left[\begin{array}{cc}
m_{1} & 0 \\
0 & m_{2}
\end{array}\right]\left[\begin{array}{l}
x_{W} \\
y_{W}
\end{array}\right]=M X
$$

The purpose of the camera calibration is to measure the value of the calibration coefficient matrix $\mathrm{M}$.

\subsection{Image show}

In the process of image programming, Image display is very important. It is always used to see whether the result is correct. So we need to write image show function. The main function we use to display images is SetDIBitsToDevice (). The function is to display the image on the output display. The function prototype is: int SetDIBitsToDevice(HDC hdc, int xDest, int Ydest, DWORD dwWidth, DWORD dwHeight, intXSrc, int Ysrc, UINT uStartScan, UINT cScanLines, CONST VOID *lpvBits, CONST BITMAPINFO *lpbmi, UINT fuColorUse).

The code of image show is as follows:

Begin

void setBitmapInfo(BITMAPINFO *bitmapInfo,int width,int height)

\{

bitmapInfo->bmiHeader.biSize = sizeof(BITMAPINFOHEADER);

bitmapInfo->bmiHeader.biWidth = width;

bitmapInfo->bmiHeader.biHeight = -height;

bitmapInfo->bmiHeader.biPlanes $=1$;

bitmapInfo->bmiHeader.biBitCount $=8$;

bitmapInfo->bmiHeader.biCompression $=0$; 


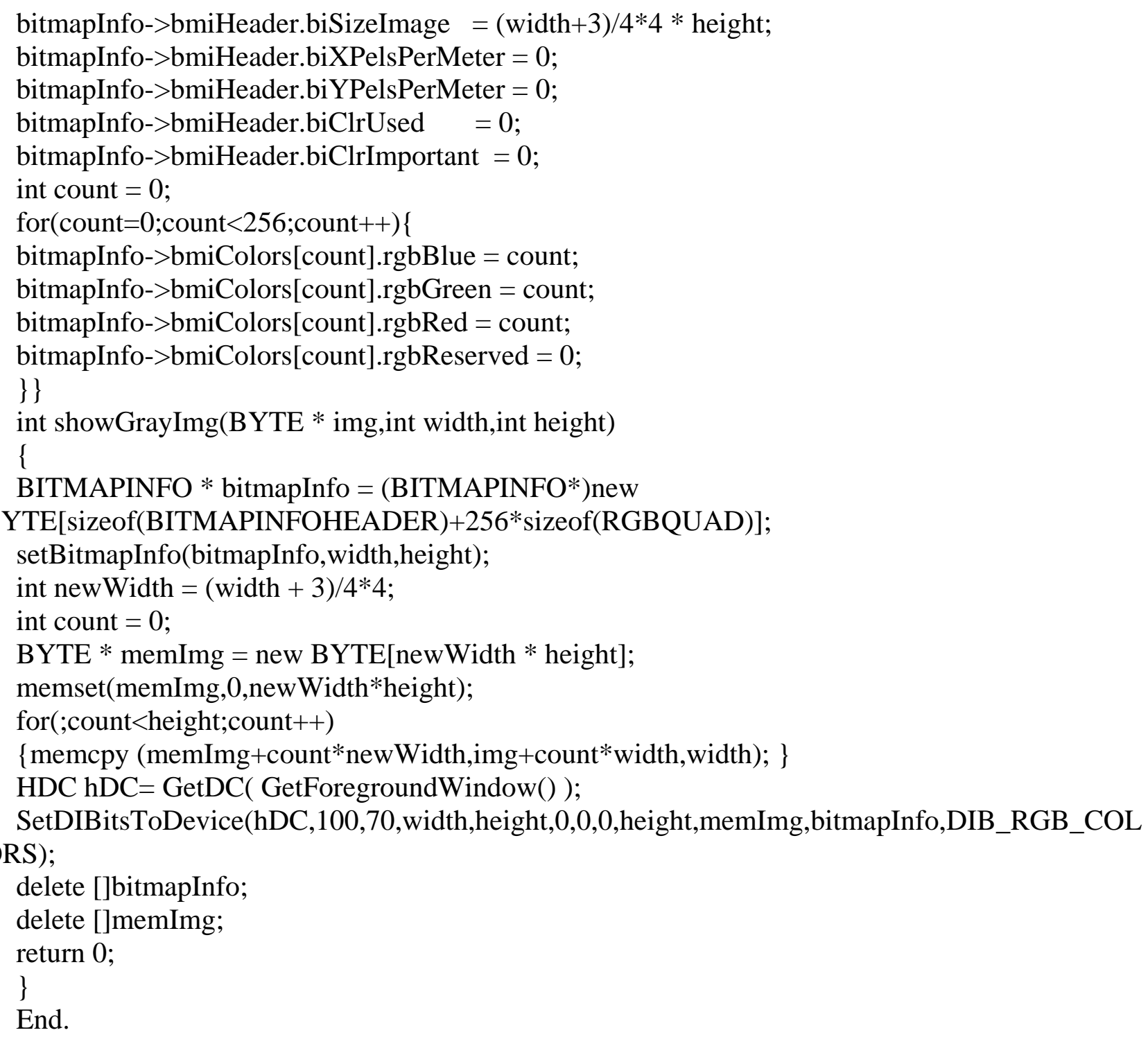

\subsection{Car seat detection}

The visual saliency mechanism is used to deal with the salient features of the colour histogram. Use image region and local RGB colour information to build saliency model, and then use the saliency model and image RGB feature to map the image pixels to decimal between 0 and 1 , that is the significant value $S(x) . S(x)$ is between 0 and 1 . When the significant value $S(x)$ is near to 1 , the corresponding pixel $\mathrm{x}$ has high probability to be the target.
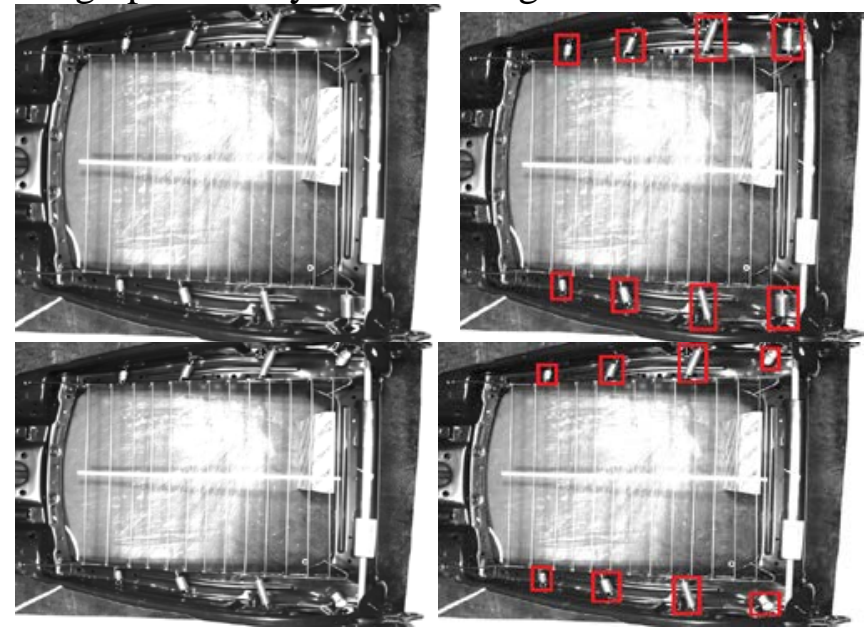

Figure 3 Target regions using significant value. 
In figure 3, the springs can be detected by significant value. The system should judge whether the car seat is qualified or not. As known to us, the springs should be connected to the side curtain. So detect the side curtain to make the judgement. Because the side curtain is always a line, so we detect the side curtain using Hough transform. Hough transform is proposed by Hough in 1962. It is used to detect curves in straight lines, circles, parabolas, ellipses and other shapes, which can be described in a certain functional relationship. It has been successfully applied in many areas, such as image analysis, pattern recognition, and so on. The basic principle of Hough transform is to transform the curves (including straight lines) in the image space to the parameter space. By detecting the extreme points in the parameter space, we can identify the description parameters of the curve, and extract the regular curves in the image.
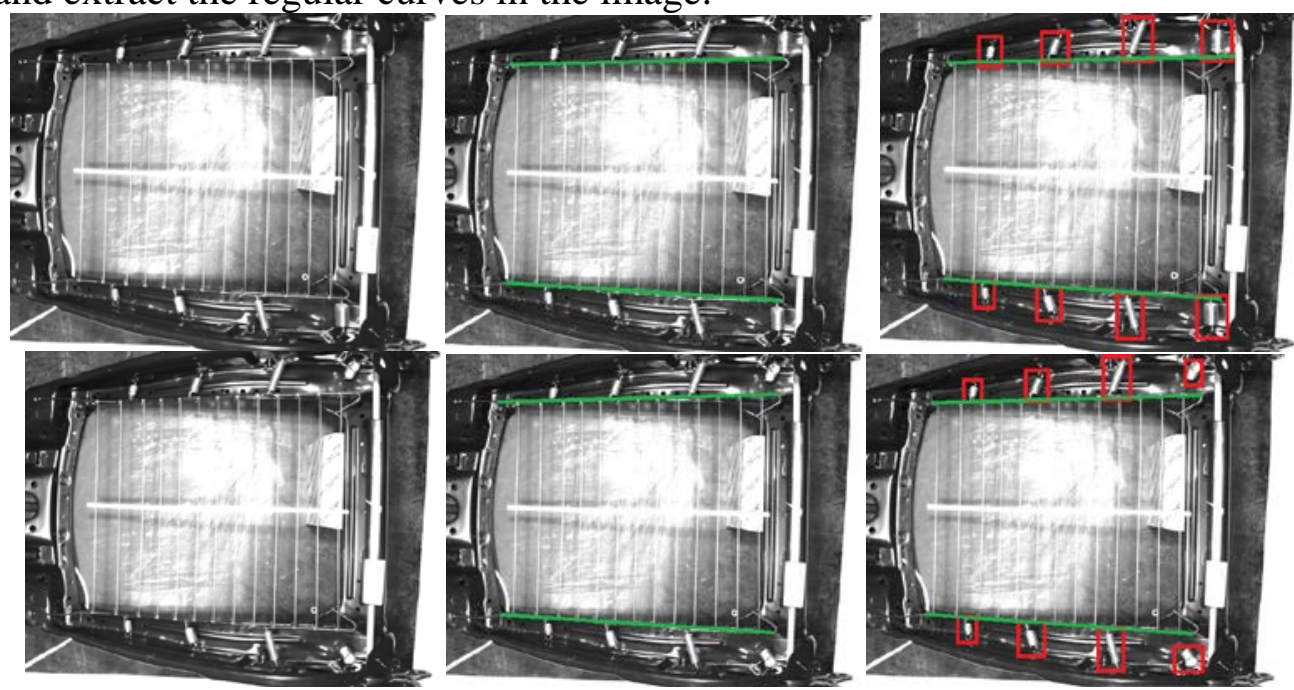

Figure 4 Detection process.

From figure 4, it can be easily seen that when there are 8 intersections between the springs and the side curtains, the car seat is qualified. When the intersections are less than 8 , it is unqualified.

\section{Conclusion}

In order to design a fast and accurate detection system to detect car seat, a real-time detection system based on vision is proposed in this paper. Firstly, give the total block diagram of the system. Secondly, give the camera calibration method. Third, use significant value to detect the springs and use the Hough transform to detect the side curtain. Calculate the intersections between springs and the side curtain. If the intersections are 8 , the car seat is qualified.

\section{Acknowledgements}

This work is supported by Development and Reform Commission in Jilin Province (No.2017C032-2).

\section{References}

[1] Danelljan M, Khan F S, Felsberg M, et al. Adaptive Color Attributes for Real-Time Visual Tracking[C]. Computer Vision and Pattern Recognition (CVPR), pp.1090-1097, Columbus, OH, 23-28 Jun. 2014. IEEE.

[2] Wang Xueying, Zhou Yue, Guo Bin, et al. Design of control terminal for auto parts inspection and production line. Journal of China Metrology University,2017,9.

[3] Feng Jinzhe, YIN Haibing. Design and implementation of Android terminal in video surveillance system. Journal of China Metrology University,2016,4.

[4] Wan Peng, Wang Hongjun. Digital modeling and analysis of auto parts production line. 
Mechanical design and manufacture. 2012(12).

[5] Wang Hongjun, the study of logistics planning technology for transmission workshop based on simulation, ICMH08, Proceedings of the 6th International Conference on Material Handling,2008.

[6] Saucy, P., Mondada, F. Open access to a mobile robot on the Internet. IEEE Robotics Automat. Mag,2000,7(1): 41-47.

[7] Digital Imaging and Communications in Medicine ( DICOM) Based Standard. National Electrical Manufacturers Association, ftp://medical.nema.org/medical/dicom,2007. 